\title{
„Die Radiologie spielt bei der Versorgung von Patienten mit Gefäßkrankheiten eine wichtige Rolle ${ }^{\text {" }}$
}

Gefäßerkrankungen sind in unserer alternden Gesellschaft weit verbreitet. Ein dabei besonders häufig vorkommendes Leiden ist die periphere arterielle Verschlusskrankheit (pAVK), in der Alltagssprache auch „Schaufensterkrankheit“ genannt. Behandelt wird diese oft mit interventionellradiologischen Methoden. Wie gut der Zugang von Betroffenen zu diesen Behandlungsmethoden ist, zeigt eine aktuelle Studie. Zur Studie, Gefäßerkrankungen im Allgemeinen und der Rolle der Qualitätssicherung für die Versorgung haben wir 3 Experten aus der Deutschen Gesellschaft für Interventionelle Radiologie und minimal-invasive Therapie (DeGIR) befragt: Professor Marcus Katoh, Chefarzt des Instituts für Diagnostische und Interventionelle Radiologie im Helios-Klinikum Krefeld, Professor Andreas Mahnken, Direktor der Klinik für Diagnostische und Interventionelle Radiologie am Universitätsklinikum Gießen und Marburg (Standort Marburg) sowie Professor Philipp Paprottka, Chefarzt der Interventionellen Radiologie am Klinikum rechts der Isar, Technische Universität München.

Professor Katoh, Gefäßerkrankungen sind in der Bevölkerung weit verbreitet. Was sind die Ursachen und Folgen dieser

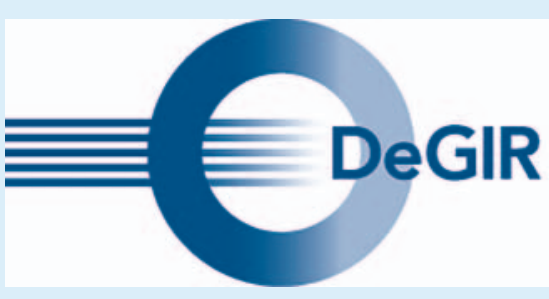

Krankheiten, etwa der peripheren arteriellen Verschlusskrankheit pAVK?

Unter der pAVK versteht man eine Erkrankung, bei der insbesondere im Bereich der Beingefäße (seltener der Armgefäße) durch Kalk- und Fettablagerungen zunehmende Engen und Verschlüsse auftreten, die dann unter Belastung, später auch in Ruhe, zu Schmerzen der betroffenen Extremität führen. In einem stark fortgeschrittenen Stadium kann auch Gewebe absterben, was dann zu so genannten Nekrosen führt. Neben einer genetischen Veranlagung spielen viele Risikofaktoren bei der Entwicklung der pAVK eine Rolle. Allen voran steht das Rauchen; aber auch Stoffwechselerkrankungen wie Diabetes, Bluthochdruck, Adipositas oder Stress begünstigen eine PAVK.

Professor Katoh, damit es nicht zu dieser Erkrankung kommt - was können Menschen präventiv dagegen tun?
Nicht Rauchen, eine gesunde Ernährung und Bewegung wären gute Rezepte, um nicht daran zu erkranken.

Welche therapeutischen Ansätze gibt es bei der Behandlung von PAVK, Herr Professor Katoh?

In einem frühen Stadium erfolgt die Therapie in erster Linie konservativ. Hierbei versucht man zunächst, die oben genannten Risikofaktoren so gut wie möglich zu reduzieren und durch ein Gehtraining die Bildung von Kollateralen anzuregen. Je nach Stadium erfolgt zusätzlich eine medikamentöse Therapie. In einem fortgeschrittenen Stadium müssen die ursächlichen Gefäßengen und Gefäßverschlüsse beseitigt werden. Das kann radiologisch-interventionell oder operativ erfolgen. Unter einem interventionellen Eingriff versteht man eine Therapie, bei der man nach einer Punktion, zum Beispiel der Leistenarterie, durch die Gefäße arbeitet. Mithilfe von schlanken Ballon-Kathetern oder so genannten Stents können von Radiologinnen und Radiologen Engen und Verschlüsse wieder durchgängig gemacht werden. Ist dies nicht mehr möglich, bleibt nur noch die operative Anlage eines Bypasses, also einer Prothese, die den Verschluss überbrückt. 
Professor Mahnken, Sie sind Erstautor der Analyse „Versorgungslage mit interventionell-radiologischer Gefäßrevaskularisation in Deutschland - eine Analyse der DeGIR-Registerdaten 2018/2019“. Was sind die Kernaussagen der Analyse?

Die Analyse der Registerdaten hat gezeigt, dass die interventionelle Radiologie deutschlandweit in allen Regionen mit für Gefäßmedizin zertifizierten interventionellen Radiologinnen und Radiologen (DeGIRStufe 2, Modul A) in der Versorgung dieser stetig steigenden Zahl von Gefäßpatientinnen und -patienten aktiv ist. In Deutschland wird also flächendeckend eine Versorgung von Gefäßpatientinnen und -patienten durch gezielt qualifizierte interventionelle Radiologinnen und Radiologen angeboten.

Professor Mahnken, wenn wir auf Ihre Analyse zurückkommen: Warum haben Sie sich in Ihrer Analyse auf die Erkrankung PAVK fokussiert?

In einer alternden Gesellschaft sind Gefäßerkrankungen - insbesondere die pAVK einer der zentralen Einflussfaktoren für Lebensqualität und Mortalität. Einer optimalen Versorgung dieser Patientinnen und Patienten kommt damit eine sehr hohe Bedeutung zu. Die Analyse der DeGIRRegisterdaten zeigt, welche zentrale Rolle die Radiologie mit ihrer einzigartigen Kombination aus diagnostischen und therapeutischen Möglichkeiten in der Versorgung dieser Patientengruppe spielt.

Wie viele Zentren und Kliniken haben in dem von Ihnen analysierten Zeitraum Daten über Eingriffe bei pAVK gemeldet und wie viele Eingriffe wurden bundesweit durchgeführt, Herr Professor Mahnken?

Es wurden 57.732 interventionelle Prozeduren zur Behandlung von Krankheiten wie der pAVK von 228 Zentren in das DeGIRQualitätsregister eingetragen. Dies ist ein sehr großer Anteil aller in Deutschland durchgeführten Behandlungen. Die Gesamtgröße dieses Therapiefeldes zeigt aber auch, dass weiterhin Bedarf besteht, weitere Zentren für die Teilnahme am DeGIR-Qualitätsregister zu gewinnen und gezielt für Gefäßmedizin zertifizierte interventionelle Radiologinnen und Radiologen auszubilden.

Professor Paprottka, die Analyse von Professor Mahnken und seinen Kollegen basiert auf Daten zu interventionellradiologischen Eingriffen bei pAVK. Diese Daten haben Kliniken gemeldet, die am DeGIR-Qualitätsregister teilnehmen. Wie viele Kliniken nehmen generell am Register teil?

Wir haben momentan 301 Kliniken, die im Register ihre Interventionen eingeben. Von diesen sind mittlerweile 181 als DeGIRoder DeGIR-/DGNR-Ausbildungszentrum zertifiziert. Aktuell werden pro Jahr rund 195000 minimal-invasive Eingriffe eingetragen, die durch interventionelle Radiologinnen und Radiologen sowie Neuroradiologinnen und Neuroradiologen durchgeführt wurden.

Professor Paprottka, das DeGIR-Qualitätsregister basiert auf einer besonderen Qualitätssicherungssoftware. Könnten Sie schildern, welche Daten mit der Software erfasst und ausgewertet werden?

Die interventionelle Radiologie hat mit dem DeGIR-Qualitätsregister die Möglichkeit, zu erfassen, wie viele Patientinnen und Patienten interventionell-radiologisch behandelt werden. In dem Register werden unter anderem die durchgeführten Interventionen, die Komplikationen und - da die Untersuchung mithilfe von Röntgenstrahlen durchgeführt wird - Dosiswerte erfasst. Hierbei dient das Register nicht nur zur Qualitätssicherung der beteiligten Zentren: Die eingegebenen Daten werden wissenschaftlich ausgewertet und können in Zukunft zum Beispiel auch für die Ausarbeitung neuer Dosisreferenzwerte im Strahlenschutz herangezogen werden. In den letzten Jahren wurden zahlreiche Auswertungen des DeGIR-Qualitätsregisters veröffentlicht und etliche weitere sind in Arbeit.

Professor Paprottka, wie helfen die an das Register gemeldeten Daten, Diagnoseund Therapiemöglichkeiten von und für Patientinnen und Patienten zu verbessern?
Das DeGIR-Qualitätsregister verbessert durch seine breite Datenbasis die Qualität der Eingriffe wesentlich, denn sie ermöglicht wissenschaftliche Erkenntnisse, die bei der Entwicklung medizinischer Leitlinien, für die Forschung und die Gesundheitspolitik wichtig sind. Insofern trägt das Register entscheidend zu einer besseren Patientenversorgung bei. Das ist unser Ziel und in dieser Richtung entwickeln wir unsere Software auch fort. So wollen wir in Zukunft zum Beispiel in unserer Software die Möglichkeit einer langfristigen Qualitätssicherung schaffen. Das ist gerade im Bereich Onkologie wichtig, in dem Patienten zum Beispiel nach 3, 6, 9 und 12 Monaten nachkontrolliert werden müssen.

\section{Über unsere Interviewpartner}

Professor Marcus Katoh ist Chefarzt des Instituts für Diagnostische und Interventionelle Radiologie im Helios-Klinikum Krefeld. Er ist Präsident der Deutschen Gesellschaft für Interventionelle Radiologie und minimal-invasive Therapie (DeGIR). Schwerpunkte seiner Präsidentschaft bei der DeGIR sind unter anderem die Förderung des interventionsradiologischen Nachwuchses und das Voranbringen digitaler Projekte.

Professor Andreas Mahnken ist Professor für Radiologie der Philipps-Universität Marburg und Direktor der Klinik für Diagnostische und Interventionelle Radiologie am Universitätsklinikum Gießen und Marburg am Standort Marburg. Professor Mahnken ist Mitglied im Vorstand der DeGIR und President-elect.

Professor Paprottka ist Chefarzt und Universitätsprofessor für Interventionelle Radiologie am Klinikum rechts der Isar an der Technischen Universität München. Er ist seit Mai 2018 Vorstandsmitglied der DeGIR und Leiter der DeGIR-Qualitätssicherungs-Softwarelenkungsgruppe. 